\title{
Impossibility of achieving Landauer's bound for almost every quantum state
}

\author{
Paul M. Riechers $\oplus^{1,2,{ }^{*}}$ and Mile $\mathrm{Gu}^{1,2,3, \dagger}$ \\ ${ }^{1}$ Nanyang Quantum Hub, School of Physical and Mathematical Sciences, Nanyang Technological University, 637371 Singapore \\ ${ }^{2}$ Complexity Institute, Nanyang Technological University, 637335 Singapore \\ ${ }^{3}$ Centre for Quantum Technologies, National University of Singapore, 3 Science Drive 2, 117543 Singapore
}

(Received 8 March 2021; accepted 15 June 2021; published 19 July 2021)

\begin{abstract}
The thermodynamic cost of resetting an arbitrary initial state to a particular desired state is lower bounded by Landauer's bound. However, here we demonstrate that this lower bound is necessarily unachievable for every initial state (except possibly the single minimally dissipative input) for any reliable reset mechanism. Since local heating threatens rapid decoherence, this issue is of substantial importance beyond mere energy efficiency. For the case of qubit reset, we find the minimally dissipative state analytically for any reliable reset protocol, in terms of the entropy-flow vector introduced here. This allows us to verify a recent theorem about initial-state dependence of entropy production for any finite-time transformation, as it pertains to quantum state preparation.
\end{abstract}

DOI: 10.1103/PhysRevA.104.012214

\section{INTRODUCTION}

Whether initializing a quantum computer or a quantum experiment, a desired quantum state must be prepared. The thermodynamic cost of erasing the pre-existing state and replacing it with a newly prepared state is typically associated with Landauer's bound: the accepted exchange rate between information and energy [1,2]. Indeed, Landauer's bound is one of the key results tying quantum information to physical predictions via the universality of thermodynamics. In the simplest quantum version of this bound, the expected heat $Q$ released to the environment must exceed the reduction in von Neumann entropy of the system (multiplied by the thermal energy $k_{\mathrm{B}} T$ of the environment) [3,4]. It is generally accepted that in the limit of quasistatically slow transformations, the expected heat can approach Landauer's lower bound $[5,6]$.

However, in the following, we demonstrate that no reliable protocol for preparing a quantum state can achieve Landauer's bound for any more than, at best, one of infinitely many possible inputs to the preparation device. In particular, when resetting a quantum state via any reliable mechanism, we find that there is only a single input state $\alpha_{0}$ leading to minimal entropy production (which is generically a mixed state) among the uncountably infinite number of possible input states.

Suppose, for example, that a quantum state is reset in finite time in an environment of ambient temperature $T$, and assume the validity of the second law of thermodynamics. Then, even if the reset mechanism approaches Landauer's bound for the

\footnotetext{
*pmriechers@gmail.com

${ }^{\dagger}$ mgu@quantumcomplexity.org
}

Published by the American Physical Society under the terms of the Creative Commons Attribution 4.0 International license. Further distribution of this work must maintain attribution to the author(s) and the published article's title, journal citation, and DOI. minimally dissipative input, the reset will necessarily produce more than Landauer's required heat $Q_{\text {Landauer }}$ for every other input. In particular, for any input $\rho_{0}$ to any reliable erasure protocol

$$
Q-Q_{\text {Landauer }} \geqslant k_{\mathrm{B}} T D\left[\rho_{0} \| \alpha_{0}\right],
$$

where $D\left[\rho_{0} \| \alpha_{0}\right]$ is the quantum relative entropy between the actual input $\rho_{0}$ and the minimally dissipative input $\alpha_{0}$.

In the following, we demonstrate the predictive power of this result and unravel its important implications for quantum state preparation. To get there, Sec. II first reviews and further develops the relevant theory, leading to a generalization of Eq. (1), which applies to finite-time transformations of any physical system placed in any environment. Section III points out the immediate consequences. To address the thermodynamic cost of initializing any quantum computing algorithm, we then develop further analytic results for qubit erasure in Sec. IV. We find the exact minimally dissipative quantum state analytically for any reliable qubit-reset protocol, in terms of the entropy-flow vector introduced here. The entropy-flow vector, in turn, is found algebraically for any qubit transformation protocol, via experimentally obtained heats, from any four linearly independent initial states. In Sec. V, we demonstrate our results with explicit physical models for qubit erasure. This allows us to verify the recent theoretical results for initial-state dependence of entropy production [Eq. (9)], developed for any finite-time transformation in Ref. [7], as applied to quantum state preparation. Finally, we examine the thermodynamic penalty of decoherence, and show that this bounded contribution from decoherence is often overshadowed by the more dire penalty imposed by the purity of the minimally dissipative input state.

The unachievability of Landauer's bound has been explored from several other directions before. For example, the authors of Ref. [4] showed that finite-dimensional quantum baths can never exactly achieve Landauer's bound, although Landauer's bound can be approached in the limit of large 
baths. In addition, in the single-shot regime, the independentand-identically-distributed (IID) limit of many coexisting copies of the system is necessary to reliably approach Landauer's bound in a single shot (rather than just on average) $[8,9]$. However, our result is even more severe than these previously acknowledged limitations of Landauer's bound. Even in the case of large baths, and when concerned with only average quantities, we find that Landauer's bound still cannot be approached by more than a single initial quantum state for any reliable implementation of reset.

\section{THERMODYNAMICS OF QUANTUM RESET}

\section{A. Background}

Nonequilibrium thermodynamics is largely a theory of entropy production. For example, the second law of thermodynamics states that entropy production is expected to be nonnegative for any thermodynamic process, from any initial state $\rho_{0}$ :

$$
\Sigma_{\rho_{0}} \geqslant 0 \text {. }
$$

The validity of the second law requires that the system is initially uncorrelated with the environment, and each part of the environment is initially uncorrelated and in local equilibrium $[4,7,10]$. This means that the initial joint state of system and environment is a product state $\rho_{0}^{\text {tot }}=\rho_{0} \otimes \rho_{0}^{\text {env }}$ with $\rho_{0}^{\text {env }}=$ $\bigotimes_{b \in \mathbb{B}} \pi_{0}^{(b)}$, where $\pi_{0}^{(b)}$ is a state of local equilibrium for bath $b$. However, the correlation between the system and baths, as well as the correlation among baths, generically builds up over time, while the baths also generically depart from local equilibrium [11].

The thermodynamic process can be made explicit through the control protocol $x_{0: \tau}$ which implies the trajectory of the joint system-baths Hamiltonian $H_{x_{t}}^{\text {tot }}$ from time 0 through $\tau$. The control protocol induces a net time evolution $\mathcal{U}_{x_{0: \tau}}$ of the system-baths supersystem, so that the system's state at the end of the transformation is

$$
\rho_{\tau}=\Gamma\left(\rho_{0}\right)=\operatorname{tr}_{\mathrm{env}}\left(\mathcal{U}_{x_{0: \tau}} \rho_{0} \otimes \rho_{0}^{\mathrm{env}} \mathcal{U}_{x_{0: \tau}}^{\dagger}\right)
$$

In general, $x_{t}$ may consist of any number of parameters. Different thermodynamic processes correspond to different control protocols.

In the quantum setting, the expected entropy production (from time 0 to $\tau$ ) is the expected entropy flow $\boldsymbol{\Phi}_{\rho_{0}}$ to the environment beyond any compensating reduction in the von Neumann entropy of the system $S\left(\rho_{t}\right)=-\operatorname{tr}\left(\rho_{t} \ln \rho_{t}\right)$ :

$$
\boldsymbol{\Sigma}_{\rho_{0}}=\boldsymbol{\Phi}_{\rho_{0}}+k_{\mathrm{B}} S\left(\rho_{\tau}\right)-k_{\mathrm{B}} S\left(\rho_{0}\right),
$$

where $k_{\mathrm{B}}$ is Boltzmann's constant.

Entropy flow has been central to thermodynamics since the discoveries of Clausius, when he found that dividing heat by the absolute temperature of each bath allowed a precise statement of the second law of thermodynamics [12]. Dividing heat by the temperature of the bath gives an Aequivalenzwerth or "equivalence-value" of the usefulness of that heat for affecting change [12]. More generally, the flow of energy, particles, volume, and so on, all have an equivalence-value to affect change; and entropy flow sums up the equivalence-value of environmental resources consumed during a transformation.
For general thermodynamic transformations, we find that the expected entropy flow can be represented as

$$
\boldsymbol{\Phi}_{\rho_{0}}:=-k_{\mathrm{B}} \int_{0}^{\tau} \operatorname{tr}\left(\dot{\rho}_{t}^{\mathrm{env}} \ln \pi_{t}^{\mathrm{env}}\right) d t,
$$

where $\rho_{t}^{\text {env }}$ is the reduced state of the environment at time $t$. The reference state $\pi_{t}^{\text {env }}$ always represents the environment as a set of thermodynamic baths $\mathbb{B}$ in local equilibrium: $\boldsymbol{\pi}_{t}^{\text {env }}=\bigotimes_{b \in \mathbb{B}} \boldsymbol{\pi}_{t}^{(b)}$. To encompass a very broad class of physical scenarios, we allow the reference state to depend on time. However, we assume in the following that the reference state is independent of the initial state of the system.

For example, if each bath has a time-independent grand canonical reference state with temperature $T^{(b)}$ and chemical potentials $\left\{\mu^{(b, \ell)}\right\}_{\ell}$, then the expected entropy flow takes on the familiar form $[10,11,13,14]$

$$
\boldsymbol{\Phi}_{\rho_{0}}=\sum_{b \in \mathbb{B}} \frac{Q^{(b)}}{T^{(b)}}-\frac{1}{T^{(b)}} \sum_{\ell} \mu^{(b, \ell)} \Delta\left\langle N^{(b, \ell)}\right\rangle,
$$

where the heat $Q^{(b)}=\Delta \operatorname{tr}\left(\rho_{t}^{(b)} H^{(b)}\right)$ is the expected energy change of bath $b$ over the course of the process and $\Delta\left\langle N^{(b, \ell)}\right\rangle=\Delta \operatorname{tr}\left(\rho_{t}^{(b)} N^{(b, \ell)}\right)$ is the expected change in the bath's number of $\ell$-type particles. Here $\rho_{t}^{(b)}$ is the reduced state of bath $b$ at time $t$. Equation (6) was used to explore entropy production even in the case of arbitrarily small baths, by fixing the reference state via the initial temperatures and chemical potentials $[10,11]$.

Equations (2) and (4) together immediately imply a very general form of Landauer's bound

$$
\boldsymbol{\Phi}_{\rho_{0}} \geqslant k_{\mathrm{B}} S\left(\rho_{0}\right)-k_{\mathrm{B}} S\left(\rho_{\tau}\right) \text {. }
$$

In other words, whenever the second law of thermodynamics is valid, the change in the system's entropy bounds the expected entropy flow.

If there is a single thermal bath at temperature $T$, then the expected entropy flow is simply

$$
\boldsymbol{\Phi}_{\rho_{0}}=Q / T
$$

where $Q$ denotes the expected heat flow to the bath, i.e., its change in energy. Landauer's bound for the heat released to the environment during reset is then $Q \geqslant Q_{\text {Landauer }}$ with $Q_{\text {Landauer }}:=k_{\mathrm{B}} T\left[S\left(\rho_{0}\right)-S\left(\rho_{\tau}\right)\right]$. Paradigmatic "bit erasure" takes a completely mixed state $\rho_{0}=I / 2$ to a pure state $\rho_{\tau}=$ $|0\rangle\langle 0|$, yielding the $S\left(\rho_{0}\right)-S\left(\rho_{\tau}\right)=\ln 2$ that leads to the most familiar form of Landauer's bound

$$
Q \geqslant k_{\mathrm{B}} T \ln 2
$$

for the heat required to erase either a bit or a qubit.

Entropy production quantifies the heat (or entropy flow, more generally) produced beyond Landauer's bound. Positive entropy production implies thermodynamic irreversibility: an effectively irreversible loss of thermodynamic resources. The cause of this effective irreversibility is easier to understand when we decompose the expected entropy production into a collection of nonnegative contributions, as in Ref. [10]. The decomposition shows that the nonnegativity of entropy production can be attributed to both (1) the growth of total correlation among the system and all baths and (2) the nonequilibrium addition to free energy built up in each bath. 
Both of these structural features are assumed to be too difficult to practically leverage, which is the reason for the effective loss of useful resources.

For any thermodynamic process that implements a nonunitary transformation of the system, the expected entropy production from time 0 through $\tau$ will depend on the initial state of the system. It is useful to consider any minimally dissipative state

$$
\alpha_{0} \in \operatorname{argmin}_{\rho_{0}} \boldsymbol{\Sigma}_{\rho_{0}} .
$$

In Ref. [7], we recently established that the expected entropy production (from any initial state $\rho_{0}$ that acts on the support of $\left.\alpha_{0}\right)$ is quantified by

$$
\boldsymbol{\Sigma}_{\rho_{0}}-\boldsymbol{\Sigma}_{\alpha_{0}}=k_{\mathrm{B}} D\left[\rho_{0} \| \alpha_{0}\right]-k_{\mathrm{B}} D\left[\rho_{\tau} \| \alpha_{\tau}\right],
$$

where $D[\rho \| \alpha]=\operatorname{tr}(\rho \ln \rho)-\operatorname{tr}(\rho \ln \alpha)$ is the quantum relative entropy. This generalizes the classical result found in Ref. [15], where the quantum relative entropy between quantum states reduces to the Kullback-Leibler divergence between classical distributions. Appendix A further extends the generality of this result, proving that it remains valid even when the environmental reference state $\pi_{t}^{\text {env }}$ is time dependent. Equation (9) is very general and is valid in the presence of arbitrary initial environments, even when the second law is not. (The examples to follow, however, all fall within the purview of the second law.)

\section{B. Consequences of reliability and precision}

In the following, we focus on reliable reset protocols, which induce the net transformation

$$
\Gamma\left(\rho_{0}\right) \approx r_{\tau}
$$

to the input-independent state $r_{\tau}$. Reliability can be quantified by the maximal trace distance between the final and desired state, $\epsilon=\sup _{\rho_{0}} \frac{1}{2}\left\|\Gamma\left(\rho_{0}\right)-r_{\tau}\right\|_{1}$, which should be very nearly zero. The following results therefore apply not only to erasing a qubit but also to many other important scenarios, including the following: (1) to initialize any number of registers of a quantum computer; (2) to produce a maximally mixed state; (3) to achieve equilibrium; (4) to establish a nonequilibrium steady state; and (5) to prepare a special state like a Bell state.

In the $\epsilon \rightarrow 0$ limit of high-fidelity reset, $\rho_{\tau}$ and $\alpha_{\tau}$ both approach $r_{\tau}$, and so $D\left[\rho_{\tau} \| \alpha_{\tau}\right] \rightarrow 0$ when reliably resetting to a mixed state [7]. In this limit of reliable reset, the difference in expected entropy production from any initial density matrix $\rho_{0}$ defined on the support of $\alpha_{0}$ is then exactly proportional to the initial distinguishability between $\rho_{0}$ and $\alpha_{0}$, as quantified by the quantum relative entropy

$$
\boldsymbol{\Sigma}_{\rho_{0}}=\boldsymbol{\Sigma}_{\alpha_{0}}+k_{\mathrm{B}} D\left[\rho_{0} \| \alpha_{0}\right]
$$

As the desired final state approaches a pure state, or a state with limited support, Eq. (11) remains valid provided that the final states all converge with sufficient precision. In particular, we can quantify the precision of final states via half the maximal trace distance between final states: $\epsilon^{\prime}=$ $\sup _{\rho_{0}, \rho_{0}^{\prime}} \frac{1}{4}\left\|\Gamma\left(\rho_{0}\right)-\Gamma\left(\rho_{0}^{\prime}\right)\right\|_{1}$. By the triangle inequality, reliability guarantees precision: $\epsilon^{\prime} \leqslant \epsilon$. While it is always true that $0 \leqslant D\left[\rho_{\tau} \| \alpha_{\tau}\right] \leqslant D\left[\rho_{0} \| \alpha_{0}\right]$, we adapt the theorems found in Ref. [16] to derive a number of stricter bounds implied by the precision and reliability of reset protocols. For example, we find

$$
D\left[\rho_{\tau} \| \alpha_{\tau}\right] \leqslant 4 \epsilon^{\prime} \ln \left(\frac{d}{4 \epsilon^{\prime} \sqrt{s}}\right) \leqslant 4 \epsilon \ln \left(\frac{d}{4 \epsilon \sqrt{s}}\right),
$$

where $d$ is the dimension of the Hilbert space and $s$ is $\alpha_{\tau}$ 's smallest eigenvalue. Appendix B provides yet much sharper upper bounds. A useful lesson is that the final relative entropy $D\left[\rho_{\tau} \| \alpha_{\tau}\right]$ goes to zero as the minimally dissipative state approaches a pure state, provided that $\epsilon^{\prime} \leqslant s$, i.e., provided that the final states converge faster than they purify. This condition is naturally fulfilled for typical protocols that reliably reset to a pure state. In the limit of perfect reset $(\epsilon=0)$, Eq. (11) is always exactly satisfied.

In the following, we will demonstrate the strong validity and power of Eq. (11) via several explicit examples. In the first example, given in Sec. V A (where the system is thrown away and replaced with a nearly pure state from an effectively cold bath) the final state is precisely the initial bath state. As a consequence, $\epsilon^{\prime}=0$ for this example, and Eq. (11) is fulfilled exactly. Our later examples, given in Sec. V B, investigate RESET via time-dependent Lindbladian dynamics. Lindbladian dynamics imply an exponential decay of distinction among states, as all states converge towards the instantaneous steady state of the Lindblad operator. Accordingly, precision improves exponentially with time for any Lindbladian dynamics, i.e., $\epsilon^{\prime}$ decays exponentially towards 0 with time. This makes Eq. (11) an excellent approximation for RESET via Lindbladian dynamics, whenever the protocol is run for long enough to ensure reliable reset towards the desired state.

The next section explicates several important implications of Eq. (11). Subsequently, Sec. IV shows how the minimally dissipative state can be found from the entropy-flow vector, which will be introduced shortly. This transforms Eq. (11) from a neat theoretical result into a powerful predictive tool for real physical systems.

\section{OBSERVATIONS}

Our first observation is that there is always a unique minimally dissipative initial state for any reliable reset protocol. If $\alpha_{0}$ has full support, this follows from Eq. (11) and the fact that the quantum relative entropy $D\left[\rho_{0} \| \alpha_{0}\right]$ is positive unless $\rho_{0}=\alpha_{0}$. In fact, Appendix $\mathrm{C}$ guarantees that $\alpha_{0}$ has full support on the domain of states to be reset, which proves the observation.

The dissipation from most inputs grows drastically as a reset protocol is adjusted to bring the minimally dissipative state closer to a pure state. Indeed, via a well-known sensitivity of the relative entropy, the dissipation diverges for most inputs as $\alpha_{0}$ approaches a pure state. This will be demonstrated in the examples of Sec. V.

To avoid this divergent dissipation, it may be desirable to design a reset protocol to be thermodynamically optimal for the completely mixed state $\alpha_{0}=I / d$ of the $d$-dimensional quantum system. What is the heat required to reliably reset any qudit to a pure state, when the system is coupled to a single thermal bath at temperature $T$ and $\Sigma_{I / d}=0$ ? From 
Eq. (11), we observe that the heat in this case is

$$
Q=k_{\mathrm{B}} T D\left[\rho_{0} \| I / d\right]+k_{\mathrm{B}} T S\left(\rho_{0}\right)=k_{\mathrm{B}} T \ln d,
$$

independent of the initial state. Appendix $\mathrm{E}$ describes a protocol that makes the fully mixed state the minimally dissipative state. While $k_{\mathrm{B}} T \ln d$ may be reminiscent of Landauer's bound, it should be noted that this exceeds Landauer's bound of $k_{\mathrm{B}} T S\left(\rho_{0}\right)$ by $k_{\mathrm{B}} T D\left[\rho_{0} \| I / d\right]$. If the input is not completely mixed, then less heat is possible by designing the reset protocol to be optimal for the true input. However, when the true input is unknown, or if a single protocol is part of a prefabricated design meant to reset states of diverse origin, this may be an acceptable price for ignorance.

Further general insight can be gained by decomposing the quantum relative entropy into contributions from classical relative entropy and quantum coherence. The classical relative entropy involves two classical probability distributions $\mathcal{Q}_{0}$ and $\mathcal{P}_{0}$ over a complete orthonormal set of $\alpha_{0}$ 's right eigenstates. Specifically, $\mathcal{Q}_{0}(s)=\left\langle s\left|\alpha_{0}\right| s\right\rangle$ is the probability that $\alpha_{0}$ would be found in the eigenstate $|s\rangle$ if the state is projectively measured in its eigenbasis. Similarly, $\mathcal{P}_{0}(s)=\left\langle s\left|\rho_{0}\right| s\right\rangle$ is the probability that $\rho_{0}$ would appear to be in the state $|s\rangle$ if measured in the eigenbasis of $\alpha_{0}$.

It is useful to note that the quantum relative entropy can always be decomposed as [7]

$$
D\left[\rho_{0} \| \alpha_{0}\right]=D_{\mathrm{KL}}\left[\mathcal{P}_{0} \| \mathcal{Q}_{0}\right]+C_{\alpha_{0}}\left(\rho_{0}\right) .
$$

Above, $D_{\mathrm{KL}}\left[\mathcal{P}_{0} \| \mathcal{Q}_{0}\right]$ is the classical relative entropy, also known as the Kullback-Leibler divergence, which is always nonnegative. Finally, $C_{\alpha_{0}}\left(\rho_{0}\right)$ is the relative entropy of coherence [17], which quantifies the coherence of $\rho_{0}$ on the eigenbasis of $\alpha_{0}{ }^{1}$ The relative entropy of coherence is also nonnegative.

The nonnegativity of the two terms in this decomposition leads to our third observation, that any initial coherence on the minimally dissipative eigenbasis directly results in extra dissipation during state reset, compared to the same state decohered in that basis.

It is worth emphasizing that coherence relative to the minimally dissipative eigenbasis is the relevant coherent quantity leading to dissipation for general thermodynamic transformations. Crucially, this minimally dissipative eigenbasis is generally not the energy eigenbasis of the system. Nevertheless, the two bases coincide for simple thermal operations like relaxation processes, as explored in many recent studies including Refs. [18-20].

At the same time, we note that the the relative entropy of coherence is always upper bounded by a constant, $\ln d$, for a $d$-dimensional system. Hence, for any process with very large entropy production $\boldsymbol{\Sigma}_{\rho_{0}}-\boldsymbol{\Sigma}_{\alpha_{0}} \gg k_{\mathrm{B}} \ln d$, the classical limit (i.e., ignoring the possibility of coherence) will suffice to explain the bulk of the dissipation. In such cases, the Kullback-Leibler divergence is responsible for most of the dissipation, with coherence playing a relatively minor role.

\footnotetext{
${ }^{1}$ The relative entropy of coherence can be written as $C_{\alpha_{0}}\left(\rho_{0}\right)=$ $S\left(P_{0}\right)-S\left(\rho_{0}\right)$, where $P_{0}=\sum_{s \in \mathbb{E}_{\alpha_{0}}} \mathcal{P}_{0}(s)|s\rangle\langle s|$ is the initial state decohered in $\alpha_{0}$ 's eigenbasis $\mathbb{E}_{\alpha_{0}}$.
}

With the general theoretical framework laid out, we can now circle back to our opening comments. Whenever the second law is valid, the nonnegativity of $\boldsymbol{\Sigma}_{\alpha_{0}}$ together with Eq. (11) imply that

$$
\boldsymbol{\Sigma}_{\rho_{0}} \geqslant k_{\mathrm{B}} D\left[\rho_{0} \| \alpha_{0}\right]
$$

for any reliable reset protocol. This generalization of Eq. (1) is necessary when heat does not capture all aspects of entropy flow. It should be noted, however, that Eq. (11) is much more predictive than Eq. (13) since the former is an equality in the limit of reliable reset.

In the next section, we introduce the entropy-flow vector, and identify the minimally dissipative state for any process that reliably resets a qubit. This then allows us to draw further lessons from the investigation of several examples.

\section{MINIMALLY DISSIPATIVE STATE FOR QUBIT ERASURE}

Landauer's bound is typically associated with reset of a bit or qubit to a particular computational state 0 . In the quantum setting, this corresponds to resetting the quantum state to a pure state $r_{\tau}=|0\rangle\langle 0|$.

The instantaneous state of a qubit can be fully described by its Bloch vector $\vec{a}=\left(a_{x}, a_{y}, a_{z}\right) \in\left\{\vec{r} \in \mathbb{R}^{3}:|\vec{r}| \leqslant 1\right\}$ via $\rho_{t}=\frac{1}{2}[I+\vec{a}(t) \cdot \vec{\sigma}]$, where $\vec{\sigma}=\left(\sigma_{x}, \sigma_{y}, \sigma_{z}\right)$ is the vector of Pauli matrices.

\section{A. Entropy-flow vector}

Appendix D shows that there is an input-independent entropy-flow vector $\vec{\phi}=\left(\phi_{x}, \phi_{y}, \phi_{z}\right)$ for each control protocol such that

$$
\boldsymbol{\Phi}_{\rho_{0}}=\boldsymbol{\Phi}_{I / 2}+\frac{1}{2} \vec{a} \cdot \vec{\phi}
$$

for any initial state $\rho_{0}=I / 2+\frac{1}{2} \vec{a} \cdot \vec{\sigma}$. Conveniently, the expected entropy flow from the completely mixed state $\boldsymbol{\Phi}_{I / 2}$ together with the entropy-flow vector $\vec{\phi}$ can both be inferred via simple linear algebra when the expected entropy flow is measured from any four experimentally accessible initial states.

Suppose we have access to any four linearly independent initial density matrices $\rho_{0}^{(n)}$ with Bloch vectors $\vec{a}^{(n)}$, and their resultant expected entropy flow $\boldsymbol{\Phi}_{\rho_{0}^{(n)}}$, for $n \in\{1,2,3,4\}$. Almost any four initial density matrices chosen at random would suffice since it is unlikely that a random density matrix will lie in the subspace spanned by the previously chosen density matrices. As a concrete, and likely useful example, we could choose the four initial states to be the completely mixed state $I / 2$, together with the pure $\hat{x}, \hat{y}$, and $\hat{z}$ states: $\left(I+\sigma_{x}\right) / 2$, $\left(I+\sigma_{y}\right) / 2$, and $\left(I+\sigma_{z}\right) / 2$, respectively.

From Eq. (14), we have

$$
\underbrace{\left[\begin{array}{llll}
2 & a_{x}^{(1)} & a_{y}^{(1)} & a_{z}^{(1)} \\
2 & a_{x}^{(2)} & a_{y}^{(2)} & a_{z}^{(2)} \\
2 & a_{x}^{(3)} & a_{y}^{(3)} & a_{z}^{(3)} \\
2 & a_{x}^{(4)} & a_{y}^{(4)} & a_{z}^{(4)}
\end{array}\right]}_{\equiv A}\left[\begin{array}{c}
\boldsymbol{\Phi}_{I / 2} \\
\phi_{x} \\
\phi_{y} \\
\phi_{z}
\end{array}\right]=2\left[\begin{array}{c}
\boldsymbol{\Phi}_{\rho_{0}^{(1)}} \\
\boldsymbol{\Phi}_{\rho_{0}^{(2)}} \\
\boldsymbol{\Phi}_{\rho_{0}^{(3)}} \\
\boldsymbol{\Phi}_{\rho_{0}^{(4)}}
\end{array}\right] .
$$


From Eq. (15), we can obtain an expression for the entropyflow vector

$$
\left[\begin{array}{c}
\boldsymbol{\Phi}_{I / 2} \\
\vec{\phi}
\end{array}\right]=2 A^{-1}\left[\begin{array}{c}
\boldsymbol{\Phi}_{\rho_{0}^{(1)}} \\
\boldsymbol{\Phi}_{\rho_{0}^{(2)}} \\
\boldsymbol{\Phi}_{\rho_{0}^{(3)}} \\
\boldsymbol{\Phi}_{\rho_{0}^{(4)}}
\end{array}\right] .
$$

Note that the invertibility of $A$ has been assured by the linear independence of the four initial states.

Once $\boldsymbol{\Phi}_{I / 2}$ and $\vec{\phi}$ have been obtained via Eq. (16), the entropy flow can be obtained analytically (for any thermodynamic process acting on a qubit) for any initial state via Eq. (14). For any reliable erasure protocol, this also leads to an analytic expression for entropy production from any initial state.

\section{B. Analytic entropy production}

For any reliable reset protocol, the entropy of the final state is independent of the initial state. In this low- $\epsilon$ limit where $S\left(\rho_{\tau}\right)=S\left(r_{\tau}\right)$, Eq. (4) tells us that entropy production can be expressed as

$$
\boldsymbol{\Sigma}_{\rho_{0}}=\boldsymbol{\Phi}_{\rho_{0}}+k_{\mathrm{B}} S\left(r_{\tau}\right)-k_{\mathrm{B}} S\left(\rho_{0}\right) .
$$

When resetting to a pure state, the entropy of the final state vanishes and Eq. (17) further simplifies to

$$
\boldsymbol{\Sigma}_{\rho_{0}}=\boldsymbol{\Phi}_{\rho_{0}}-k_{\mathrm{B}} S\left(\rho_{0}\right) \text {. }
$$

Moreover, the entropy of a qubit only depends on its purity, via the length of its Bloch vector $a=|\vec{a}|$. This is because a qubit state always spectrally decomposes into $(1+a) / 2$ times the pure state in the direction of the Bloch vector, plus $(1-a) / 2$ times the pure state in the antipodal direction on the Bloch sphere. For an initial state with Bloch vector $\vec{a}$, the initial entropy is therefore

$$
S\left(\rho_{0}\right)=-\frac{1+a}{2} \ln \left(\frac{1+a}{2}\right)-\frac{1-a}{2} \ln \left(\frac{1-a}{2}\right) .
$$

Equations (14), (18), and (19) thus yield an analytic expression for entropy production of any reliable qubit erasure protocol via (1) the Bloch vector $\vec{a}$ of the initial state, (2) the expected entropy flow $\boldsymbol{\Phi}_{I / 2}$ from the completely mixed state, and (3) the entropy-flow vector $\vec{\phi}$ induced by the protocol.

\section{Minimally dissipative state}

We can now minimize Eq. (17) to find the minimally dissipative initial state $\alpha_{0}$ analytically in terms of the entropy-flow vector for any reliable reset protocol.

We will parametrize a generic initial density matrix $\rho_{0}$ via its Bloch vector $\vec{a}=\left(a_{x}, a_{y}, a_{z}\right)$ with magnitude $a=\left(a_{x}^{2}+\right.$ $\left.a_{y}^{2}+a_{z}^{2}\right)^{1 / 2}$. The minimally dissipative initial state $\alpha_{0}$ has the Bloch vector $\vec{a}^{*}$. Differentiating the entropy production with respect to changes in the Bloch vector, we find

$$
\frac{\partial \boldsymbol{\Sigma}_{\rho_{0}}}{\partial a_{x}}=\frac{a_{x}}{2 a} \ln \left(\frac{1+a}{1-a}\right)+\frac{1}{2} \phi_{x}
$$

and similar expressions when we take the derivative with respect to $a_{y}$ or $a_{z}$. Since $\alpha_{0}$ is a unique nonpure state, the condition of minimization requires that

$$
\left.\frac{\partial \boldsymbol{\Sigma}_{\rho_{0}}}{\partial a_{x}}\right|_{a_{x}=a_{x}^{*}}=0
$$

and so on for $a_{y}^{*}$ and $a_{z}^{*}$. This immediately leads to conditions like

$$
a_{x}^{*}=-\phi_{x} \frac{a^{*}}{\ln \left(\frac{1+a^{*}}{1-a^{*}}\right)} .
$$

Combining Eq. (22) with the corresponding expressions for $a_{y}^{*}$ and $a_{z}^{*}$ according to $\left(a_{x}^{* 2}+a_{y}^{* 2}+a_{z}^{* 2}\right)^{1 / 2}=a^{*}$ yields a condition for $a^{*}$ :

$$
\ln \left(\frac{1+a^{*}}{1-a^{*}}\right)=\sqrt{\phi_{x}^{2}+\phi_{y}^{2}+\phi_{z}^{2}}=\phi,
$$

which can be solved to obtain

$$
a^{*}=\tanh (\phi / 2) \text {. }
$$

Plugging this back into Eq. (22), we find that

$$
\vec{a}^{*}=-\tanh (\phi / 2) \hat{\phi},
$$

where $\hat{\phi}=\vec{\phi} / \phi$. This gives the Bloch vector $\vec{a}^{*}$ of the minimally dissipative initial state $\alpha_{0}$, analytically for any reliable reset protocol, in terms of the protocol's entropy-flow vector $\vec{\phi}$.

Qualitatively, we can note from Eq. (25) that the minimally dissipative Bloch vector $\vec{a}^{*}$ points in the opposite direction of the entropy-flow vector $\vec{\phi}$. This enables a reduction in heat flow. As the magnitude of the entropy-flow vector $\phi$ grows beyond two, the minimally dissipative Bloch vector converges exponentially to the edge of the Bloch sphere. However, the minimally dissipative initial state is never exactly pure for any finite $\phi$ since entropy production balances entropy flow against the growth in state uncertainty, and, evidently, a sufficiently small reduction in initial-state certainty can always outweigh the smaller potential reduction in entropy flow.

\section{EXPLICIT RESET PROTOCOLS}

\section{A. First example: Reset via SWAP}

It should be noted that our preceding results are very generally applicable and are not limited to weak interactions nor any of the other approximations that are required of the common quantum master equations often employed in quantum thermodynamics (although our results indeed apply there also). To demonstrate our result in a setting of strong interactions with an explicit finite bath, we first consider reset via a swap operation between the system and a part of an effectively cold bath.

This simple scenario corresponds to "throwing away" the old state $\rho_{0}$ and replacing it with a bath state $\gamma \approx|0\rangle\langle 0|$. We consider a bath of $N$-independent qubits, $\boldsymbol{\pi}^{(b)}=\gamma^{\otimes N}$, which are initially in canonical equilibrium at temperature $T=1 / k_{\mathrm{B}} \beta$, such that $\gamma=e^{-\beta H_{b}} / Z_{b}$. This bath of Gibbs states is a bath of nearly pure $|0\rangle\langle 0|$ states if the bath Hamiltonian for each qubit is $H_{b}=-E_{b} \sigma_{z}$ with the "effectively cold" condition that $E_{b} \gg k_{\mathrm{B}} T$. To reset the state of the system, we merely swap it with a state from the effectively cold bath. This swap operation is implemented via a unitary operation on the joint system-baths supersystem, as depicted in the following 
quantum-circuit diagram:

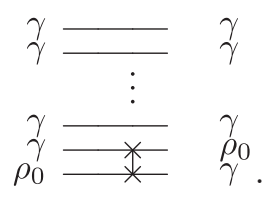

Note that entropy flow, as defined in Eq. (6), can be quantified exactly via the initial temperature of the bath, as in Refs. [10,11], for arbitrarily small environments. All thermodynamic quantities can be calculated exactly: heat, change in von Neumann entropy, and entropy production. Our calculation is valid for any Hamiltonian of the system and for any initial state $\rho_{0}{ }^{2}$

The heat transferred to the bath is

$$
\begin{aligned}
Q & =\operatorname{tr}\left(\rho_{\tau}^{(b)} H^{(b)}\right)-\operatorname{tr}\left(\rho_{0}^{(b)} H^{(b)}\right) \\
& =\operatorname{tr}\left(\rho_{0} H_{b}\right)-\operatorname{tr}\left(\gamma H_{b}\right) \\
& =k_{\mathrm{B}} T \operatorname{tr}(\gamma \ln \gamma)-k_{\mathrm{B}} T \operatorname{tr}\left(\rho_{0} \ln \gamma\right),
\end{aligned}
$$

where we used the fact that $H_{b}=-k_{\mathrm{B}} T \ln \gamma-k_{\mathrm{B}} T \ln Z_{b}$. Meanwhile, the swap operation changes the von Neumann entropy of the system $S_{\text {sys }}=S\left(\rho_{t}\right)$ according to

$$
\Delta S_{\text {sys }}=k_{\mathrm{B}} \operatorname{tr}\left(\rho_{0} \ln \rho_{0}\right)-k_{\mathrm{B}} \operatorname{tr}(\gamma \ln \gamma) .
$$

Clearly, this reset-via-swap protocol has a unique minimally dissipative initial state of $\alpha_{0}=\gamma$ since then $Q=0$ and $\Delta S_{\text {sys }}=0$ and so $\Sigma_{\alpha_{0}}=0$. For any initial state $\rho_{0}$, the entropy production will be

$$
\begin{aligned}
\Sigma_{\rho_{0}} & =Q / T+\Delta S_{\text {sys }} \\
& =k_{\mathrm{B}} D\left[\rho_{0} \| \gamma\right] .
\end{aligned}
$$

Clearly, Eq. (30) agrees with Eq. (11), which is expected since Eq. (11) quantifies the entropy production for reliable reset via any means. This and the following examples validate the more general result, but also give us the opportunity to explore the more nuanced implications.

Notably, if $\gamma \approx|0\rangle\langle 0|$, then the entropy production diverges as $\rho_{0} \rightarrow|1\rangle\langle 1|$. This points to a disadvantage of the throwaway strategy to reset. It can be thermodynamically advantageous to recycle the system state, as demonstrated in the next example where $\alpha_{0}$ can be made closer to the fully mixed state than to the desired final state.

It may be recognized that Eq. (30) has the form of a nonequilibrium addition to free energy. However, it is not related to the free energy of the system, but rather, is related to the change in nonequilibrium free energy of the bath, which is consistent with the aforementioned decomposition of entropy production. Note that Eq. (30) applies independently of the Hamiltonian of the system, so long as $\rho_{0}$ is the state of the system at the time of the swap.

\section{B. Reliable reset protocols via time-dependent Lindbladians}

We now consider the scenario of single-qubit erasure in the experimentally common regime well described by Markovian

\footnotetext{
${ }^{2}$ When the bath has many similar subsystems, the SWAP operation has a negligible effect on the overall temperature of the bath. However, Eq. (6) only requires the initial temperature to be well defined, and so does not require the large- $N$ limit.
}

master equations. Following Ref. [6], we consider a family of protocols that utilize two time-dependent control parameters $x_{t}=\left(E_{t}, \theta_{t}\right)$, which determine the time-varying Hamiltonian of the system

$$
H_{x_{t}}=\frac{E_{t}}{2}\left[\cos \left(\theta_{t}\right) \sigma_{z}+\sin \left(\theta_{t}\right) \sigma_{x}\right],
$$

where $\sigma_{x}, \sigma_{y}$, and $\sigma_{z}$ are the Pauli operators. While $E_{t}$ quantifies the energy gap between the system's instantaneous energy eigenstates, $\theta_{t}$ parametrizes the instantaneous orientation of the energy eigenbasis relative to the "computational" $z$ basis. If we assume the Markovian limit and detailed-balanced dynamics as the system interacts weakly with a large bosonic bath, then the system evolves according to the instantaneous Lindbladian [6]

$$
\begin{aligned}
\dot{\rho}_{t}=\mathcal{L}_{x_{t}}\left(\rho_{t}\right)= & \frac{i}{\hbar}\left[\rho_{t}, H_{x_{t}}\right]+\frac{c E_{t}}{\hbar}\left(N_{x_{t}}+1\right) \mathcal{D}\left[L_{x_{t}}\right]\left(\rho_{t}\right) \\
& +\frac{c E_{t}}{\hbar} N_{x_{t}} \mathcal{D}\left[L_{x_{t}}^{\dagger}\right]\left(\rho_{t}\right)
\end{aligned}
$$

where $\mathcal{D}[L](\rho)=L \rho L^{\dagger}-\frac{1}{2}\left\{L^{\dagger} L, \rho\right\}, \quad N_{x_{t}}=\left(e^{\beta E_{t}}-1\right)^{-1}$, and $c$ is the coupling strength to the bath. The time-dependent lowering operator can be represented as

$$
L_{x_{t}}=\frac{1}{2}\left[\cos \left(\theta_{t}\right) \sigma_{x}-i \sigma_{y}-\sin \left(\theta_{t}\right) \sigma_{z}\right]
$$

and satisfies the detailed balance condition $\left[L_{x_{t}}, H_{x_{t}}\right]=E_{t} L_{x_{t}}$ $[6,21]$.

This generic Hamiltonian and Lindbladian have a long history of applications to many distinct physical systems [22], and have been used recently to explore the nonequilibrium thermodynamics of qubit erasure [6].

We consider three control protocols to reset the system to the computational-basis state $|0\rangle=\sigma_{z}|0\rangle$. For all protocols, we choose the coupling strength $c=1 / 5$, the duration $\tau=50 \beta \hbar$, and the time-step for numerical integration $d t=$ $\beta \hbar / 500$.

For the first two protocols, the gap between energy eigenvalues changes smoothly as $E_{t}=E_{0}+\left(E_{\tau}-E_{0}\right) \sin ^{2}\left(\frac{\pi t}{2 \tau}\right)$ with $E_{\tau}=10 k_{\mathrm{B}} T$ and $E_{0}=E_{\tau} / 50$. During the protocol analyzed in Fig. 1, the energy eigenstates rotate according to $\theta_{t}=\pi t / \tau$. Whereas, during the alternative protocol analyzed in Fig. 2, the energy eigenstates have a fixed orientation according to $\theta_{t}=\pi$. These first two protocols are very similar to Ref. [6], but with slightly different parameter settings, and analyzed for different purposes.

The final protocol, shown in Fig. 3, achieves reset simply via relaxation to an equilibrium state which (via a large energy gap of $10 k_{\mathrm{B}} T$ ) is designed to be very close to a pure state.

In each of Figs. 1, 2, and 3, we show the evolution of five randomly sampled initial density matrices via the evolution of their Bloch vectors. These are the first three panels of each figure. The next three panels of each figure show the evolution of expected heat, system entropy, and expected entropy production that ensues from each of these five randomly sampled initial conditions. Within each figure, each initial state maintains its color and line-style across these six panels of time-series data. The wide distribution in expected heat and expected entropy production is evident for each protocol.

For each protocol, there is a single minimally dissipative initial state. Our examples demonstrate that, in general, this is 

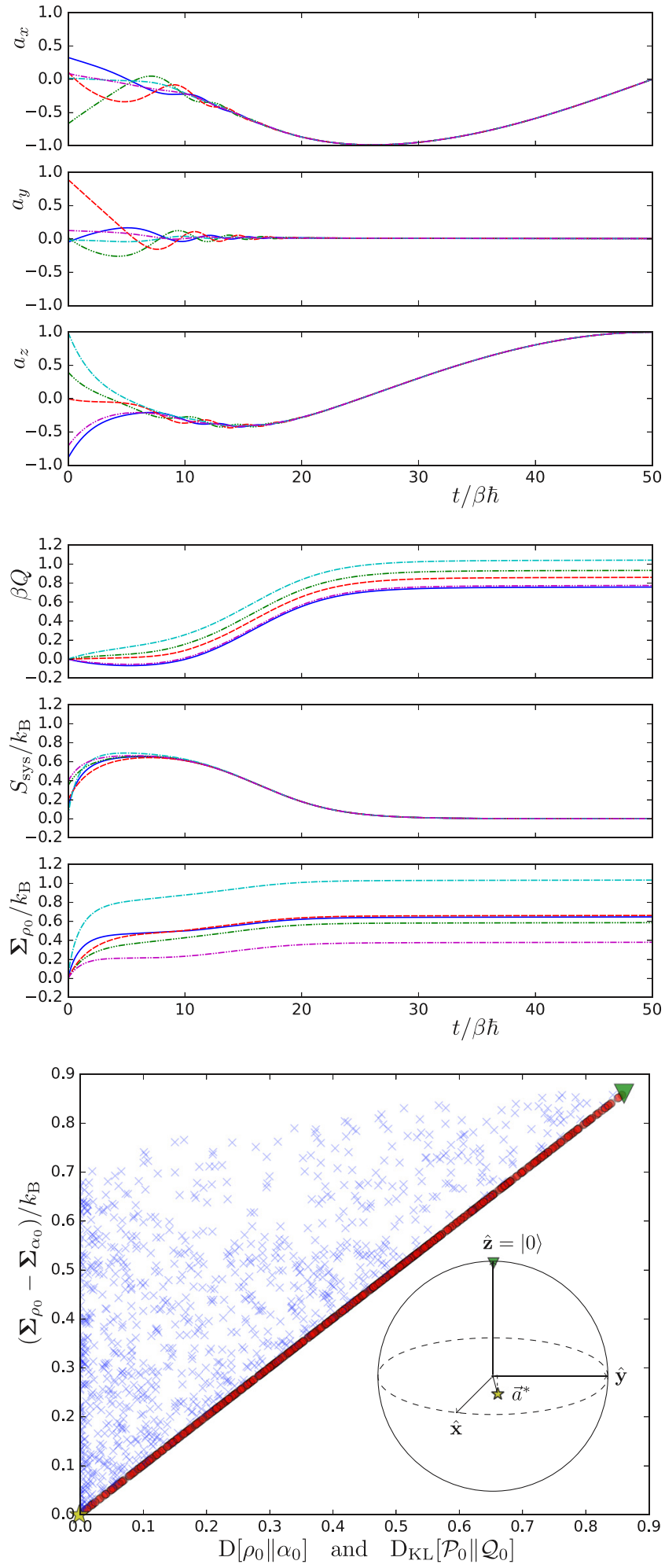

FIG. 1. A reliable protocol for erasing a qubit, which evolves the energy gap and orientation of the energy eigenstates via $E_{t}=$ $E_{0}+\left(E_{\tau}-E_{0}\right) \sin ^{2}(\pi t / 2 \tau)$ and $\theta_{t}=\pi t / \tau$, respectively. Top three panels: Evolution of the Bloch vector for five randomly sampled initial states. Next three panels: Evolution of thermodynamic quantities for the same five initial states. Bottom panel: Dissipation for 1000 random initial states vs $D\left[\rho_{0} \| \alpha_{0}\right]$ (red circles) and vs $D_{\mathrm{KL}}\left[\mathcal{P}_{0} \| \mathcal{Q}_{0}\right]$ (blue crosses).
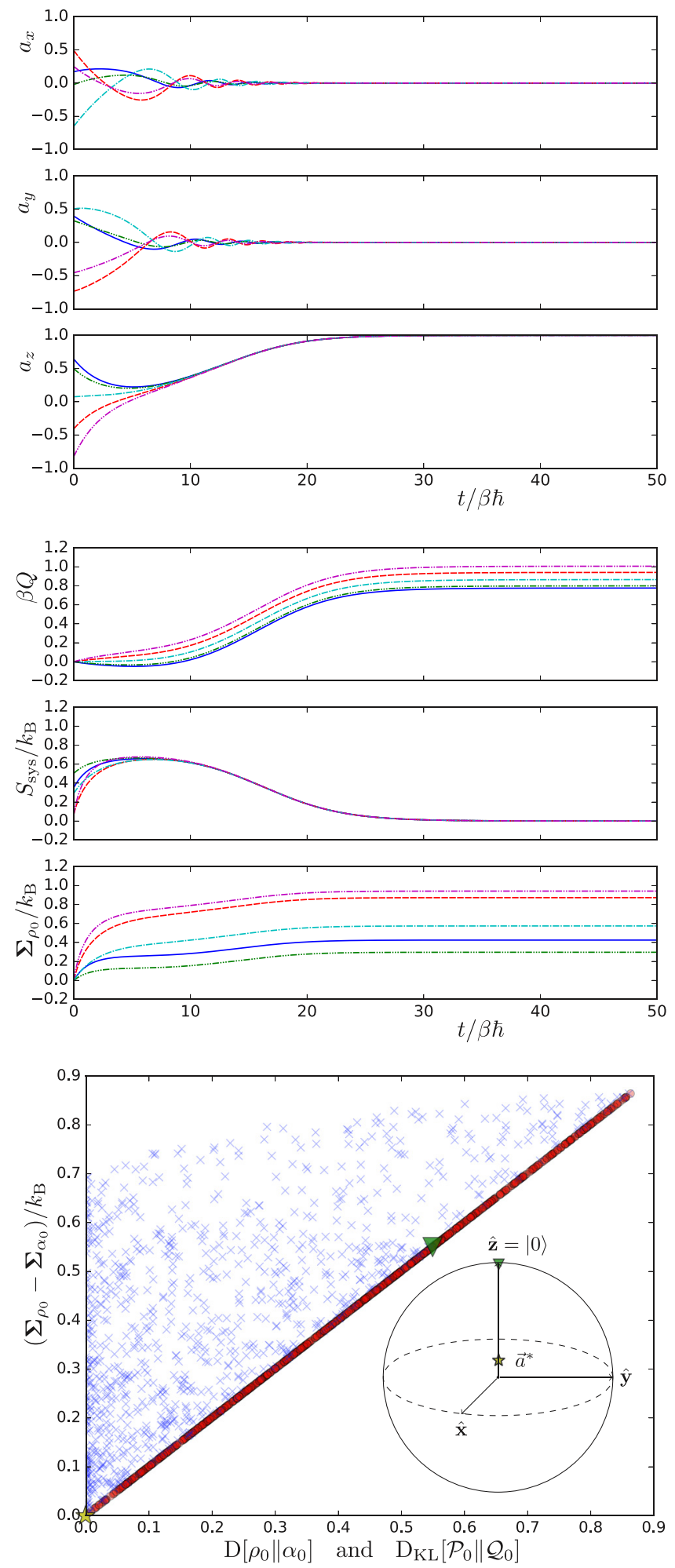

FIG. 2. A reliable protocol for erasing a qubit, which evolves the energy gap via $E_{t}=E_{0}+\left(E_{\tau}-E_{0}\right) \sin ^{2}(\pi t / 2 \tau)$ while the orientation of the energy eigenstates is fixed via $\theta_{t}=\pi$. Top three panels: Evolution of the Bloch vector for five randomly sampled initial states. Next three panels: Evolution of thermodynamic quantities for the same five initial states. Bottom panel: Dissipation for 1000 random initial states, vs $D\left[\rho_{0} \| \alpha_{0}\right]$ (red circles) and vs $D_{\mathrm{KL}}\left[\mathcal{P}_{0} \| \mathcal{Q}_{0}\right]$ (blue crosses). 

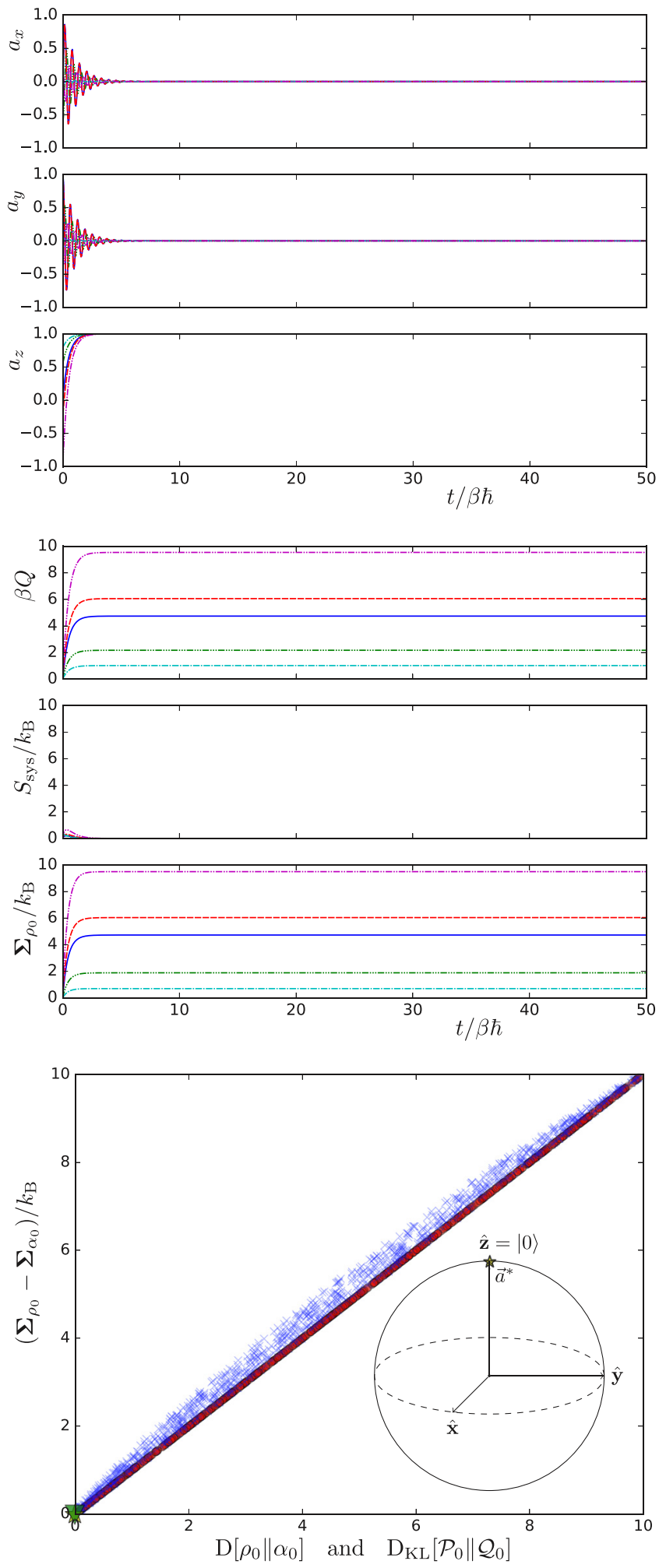

FIG. 3. A reliable protocol for erasing a qubit, which achieves reset via relaxation to thermal equilibrium via fixed energy eigenstates with $E_{t}=10 k_{\mathrm{B}} T$ and $\theta_{t}=\pi$ for $0<t \leqslant \tau$. Top three panels: Evolution of the Bloch vector for five randomly sampled initial states. Next three panels: Evolution of thermodynamic quantities for the same five initial states. Bottom panel: Dissipation for 1000 random initial states, vs $D\left[\rho_{0} \| \alpha_{0}\right]$ (red circles) and vs $D_{\mathrm{KL}}\left[\mathcal{P}_{0} \| \mathcal{Q}_{0}\right]$ (blue crosses). neither the initial equilibrium state nor the desired final state. This is most apparent for the first protocol since the minimally dissipative state is not diagonalized in the computational basis that diagonalizes both the initial equilibrium state and the desired reset state. (To see this, note that $\vec{a}^{*}$ is not along the $\hat{\mathbf{z}}$ axis in the bottom panel of Fig. 1.) Moreover, this shows that the minimally dissipative basis is, in general, distinct from the energy eigenbasis.

For each quantum erasure protocol, we find the unique minimally dissipative initial density matrix $\alpha_{0}$ via Eqs. (16) and (25), from the entropy flow observed from four of the random initial conditions. In the bottom panel of each figure, dissipation versus the quantum relative entropy $\mathrm{D}\left[\rho_{0} \| \alpha_{0}\right]$ is shown in red circles for 1000 random initial states. The red circles all lie along the identity, which clearly verifies Eq. (11), showing that $\Sigma_{\rho_{0}}-\Sigma_{\alpha_{0}}=k_{\mathrm{B}} D\left[\rho_{0} \| \alpha_{0}\right]$.

For the same 1000 random initial conditions for each figure, the off-diagonal positions of the blue crosses demonstrate the insufficiency of the classical approximation, which would use the Kullback-Leibler divergence rather than the quantum relative entropy. Via Eq. (12), the horizontal gap between $D\left[\rho_{0} \| \alpha_{0}\right]$ (red circles) and $D_{\mathrm{KL}}\left[\mathcal{P}_{0} \| \mathcal{Q}_{0}\right]$ (blue crosses) quantifies the initial coherence $C_{\alpha_{0}}\left(\rho_{0}\right)$ for each of the 1000 random initial conditions in each figure. All of this initial coherence, relative to the eigenbasis of the minimally dissipative input $\alpha_{0}$, directly leads to dissipation during RESET.

In the same bottom panels, we show the position of $\alpha_{0}$ in the Bloch sphere via its Bloch vector $\vec{a}^{*}$. Finally, the green triangle along the identity shows the dissipation incurred when the initial state is the desired final state. Starting where you end is not typically optimal; indeed, Fig. 1 shows that this is nearly the worst way to start for that protocol.

While the distribution of thermodynamic quantities appears similar for the first two protocols, we emphasize that the protocols are efficient for different initial states. This is seen most immediately via the bottom panel of Figs. 1 and 2, where it is apparent that each protocol has a distinct minimally dissipative state. States closest to the respective minimally dissipative states are efficient for the respective protocols.

The typical dissipation in Fig. 3 is much larger than in the previous two cases since the minimally dissipative state is closer to a pure state. In this case, it is interesting to note that the Kullback-Leibler divergence is responsible for most of the dissipation, with coherence playing a relatively minor role. Recall that the relative entropy of coherence is always upper bounded by a constant, $\ln d$, for a $d$-dimensional system. Hence, since this reset process is capable of very large entropy production $\Sigma_{\rho_{0}} \gg k_{\mathrm{B}} \ln d=k_{\mathrm{B}} \ln 2$, the classical limit explains the bulk of the dissipation.

\section{CONCLUSION}

Landauer's bound for the heat required to reset a state is achieved in the limit of zero entropy production. However, we showed that bound to be unachievable for almost every quantum input to any thermodynamic process for reliable reset. This follows from the fact that there is a unique initial quantum state leading to minimal entropy production for each reliable reset protocol. Moreover, we demonstrated that when a reset protocol is modified to bring this minimally dissipative 
initial state closer to a pure state, entropy production (and thus also heat) diverges for all inputs that are not close to this pure state.

For the case of qubit reset, we found the minimally dissipative state analytically in terms of the entropy-flow vector, which was introduced here. We anticipate that the entropyflow vector may be a useful concept also for larger quantum systems, via a straightforward generalization of Eq. (14).

This requisite heat for RESET revises the thermodynamics of computation, for both classical and quantum computing. Commercial classical computers do not even come close to Landauer's bound; beyond the energetic cost, this excess heating has led to the end of Moore's Law and intentional slowing of computer-clock speed [23]. Our results help explain at least part of this excess heat, while pointing to ways around it. Similarly, the energy expended to manipulate qubits has been orders of magnitude above Landauer's bound for all quantum computing demonstrations so far. While we may be willing to budget significant energy for quantum computing, local on-chip heating poses a serious threat to the very viability of quantum computing. Notably, local heating introduces uncontrolled nonunitary evolution of quantum states that destroys the delicate desired coherence and superpositions necessary for a successful quantum computation. Accordingly, our results should be leveraged in the design of processes like state preparation and error correction to minimize this errant heat.

Landauer's bound is often invoked to draw physical implications from quantum information theory, via the universality of thermodynamics. Our results show that this connection is somewhat tenuous since Landauer's bound is impossible to achieve for almost every initial state for any reliable reset mechanism. Nevertheless, nonequilibrium thermodynamics offers refined information-theoretic equalities, like Eq. (9), that are valid and offer tight predictions arbitrarily far from equilibrium. Recognizing the impossibility of Landauer's bound will be especially important for designing practical quantum computers, in which quantum states must be reliably prepared while maintaining coherence through low-heat transformations.

\section{ACKNOWLEDGMENTS}

This work was supported by the Singapore Ministry of Education Tier 1 Grant No. RG146/20, the National Research Foundation of Singapore Fellowship No. NRF-NRFF201602, the National Research Foundation and L'Agence Nationale de la Recherche joint Project No. NRF2017NRFANR004 VanQuTe, and the FQXi R-710-000-146-720 Grant "Are quantum agents more energetically efficient at making predictions?" from the Foundational Questions Institute and Fetzer Franklin Fund (a donor-advised fund of Silicon Valley Community Foundation), and the Quantum Engineering Program QEP-SP3.

\section{APPENDIX A: ENTROPY FLOW AND INITIAL-STATE DEPENDENCE OF ENTROPY PRODUCTION}

The flow of energy, particles, volume, and so on all have an equivalence-value to affect change, and entropy flow sums up the equivalence-value of environmental resources consumed during a transformation. We find that the expected entropy flow can be represented most generally as

$$
\boldsymbol{\Phi}_{\rho_{0}}=-k_{\mathrm{B}} \int_{0}^{\tau} \operatorname{tr}\left(\dot{\rho}_{t}^{\mathrm{env}} \ln \pi_{t}^{\mathrm{env}}\right) d t,
$$

where $\rho_{t}^{\text {env }}$ is the reduced state of the environment at time $t$. $\pi_{t}^{\text {env }}$ is a time-dependent reference state that represents the environment as a set of thermodynamic baths $\mathbb{B}$ in local equilibrium: $\boldsymbol{\pi}_{t}^{\text {env }}=\bigotimes_{b \in \mathbb{B}} \boldsymbol{\pi}_{t}^{(b)}$. The equilibrium state $\boldsymbol{\pi}_{t}^{(b)}$ is constructed with the bath's operators [e.g., Hamiltonian $H_{x_{t}}^{(b)}$, number operators $N_{x_{t}}^{(b, \ell)}$, etc.] that correspond to its variable observable quantities (energy, particle numbers, etc.) [24,25].

For example, if the temperatures $\left\{T_{t}^{(b)}\right\}_{b \in \mathbb{B}}$ of the baths vary over the course of a protocol, and the baths can only exchange energy, then the reference equilibrium states of the baths are canonical $\left(\pi_{t}^{(b)} \propto e^{-H_{x_{t}}^{(b)} / k_{\mathrm{B}} T_{t}^{(b)}}\right)$ and Eq. (A1) reduces to the familiar expression

$$
\boldsymbol{\Phi}_{\rho_{0}}=\sum_{b \in \mathbb{B}} \int \frac{\delta Q^{(b)}}{T_{t}^{(b)}},
$$

where $\delta Q^{(b)}=\operatorname{tr}\left(\dot{\rho}_{t}^{(b)} H_{x_{t}}^{(b)}\right) d t$ is a small transfer of energy to bath $b$.

As another prominent example, if each bath has a time-independent grand canonical reference state $\left[\pi^{(b)} \propto\right.$ $e^{-\left(H_{x_{t}}^{(b)}-\mu^{(b, \ell)} N_{x_{t}}^{(b, \ell)}\right) / k_{\mathrm{B}} T^{(b)}}$ fixed by the initial temperature $T^{(b)}$ and chemical potentials $\left\{\mu^{(b, \ell)}\right\}_{\ell}$, then Eq. (A1) reduces to Eq. (6) of the main text

$$
\boldsymbol{\Phi}_{\rho_{0}}=\sum_{b \in \mathbb{B}} \frac{Q^{(b)}}{T^{(b)}}-\frac{1}{T^{(b)}} \sum_{\ell} \mu^{(b, \ell)} \Delta\left\langle N^{(b, \ell)}\right\rangle .
$$

In some thermodynamic frameworks, the environmental reference state $\pi_{t}^{\text {env }}$ in Eq. (A1) may be time dependent. We will assume that, if $\boldsymbol{\pi}_{t}^{\text {env }}$ is time dependent, it is nevertheless (at least approximately) independent of the initial state of the system. Under this assumption, the expected entropy flow is a linear function of the initial state of the system. This linearity is evident once we write out the reduced state of the environment as $\rho_{t}^{\text {env }}=\operatorname{tr}_{\text {sys }}\left(U_{t} \rho_{0} \otimes \rho_{0}^{\text {env }} U_{t}^{\dagger}\right)$. Because the expected entropy flow is a linear function of the initial state of the system, Theorem 2 of Ref. [7] guarantees that the expected entropy production from any initial state $\rho_{0}$ (defined on the support of $\alpha_{0}$ ) is quantified by

$$
\boldsymbol{\Sigma}_{\rho_{0}}-\boldsymbol{\Sigma}_{\alpha_{0}}=k_{\mathrm{B}} D\left[\rho_{0} \| \alpha_{0}\right]-k_{\mathrm{B}} D\left[\rho_{\tau} \| \alpha_{\tau}\right],
$$

where

$$
\alpha_{0} \in \operatorname{argmin}_{\rho_{0}} \boldsymbol{\Sigma}_{\rho_{0}} .
$$

This generalizes the main result of Ref. [7] to allow for a timedependent environmental reference state. This establishes, for example, that Eq. (A3) remains valid in scenarios where the temperature profile of a bath changes through time.

\section{APPENDIX B: PRECISE RESET BOUNDS RELATIVE ENTROPY}

For any process whatsoever, $0 \leqslant D\left[\rho_{\tau} \| \alpha_{\tau}\right] \leqslant D\left[\rho_{0} \| \alpha_{0}\right]$. But we can also derive a number of stricter bounds on the final distinguishability $D\left[\rho_{\tau} \| \alpha_{\tau}\right]$ implied by precision and reliability of reset protocols. 
Recall that the trace distance $\mathcal{T}(\rho, \sigma)$ between two quantum states $\rho$ and $\sigma$ is given by $\mathcal{T}(\rho, \sigma):=\frac{1}{2}\|\rho-\sigma\|_{1}$, where $\|A\|_{1}:=\operatorname{tr}\left(\sqrt{A^{\dagger} A}\right)$ is the trace norm.

We find that the precision of final states strongly upper bounds the relative entropies among them. Recall that precision is quantified by: $\epsilon^{\prime}=\sup _{\rho_{0}, \rho_{0}^{\prime}} \frac{1}{4}\left\|\Gamma\left(\rho_{0}\right)-\Gamma\left(\rho_{0}^{\prime}\right)\right\|_{1}$. This implies

$$
\mathcal{T}\left(\rho_{\tau}, \rho_{\tau}^{\prime}\right) \leqslant 2 \epsilon^{\prime} \text { for all } \rho_{0}, \rho_{0}^{\prime} .
$$

Reliablity gives weaker bounds. Reliability is quantified by the error tolerance $\epsilon$ associated with a control protocol $\epsilon=$ $\sup _{\rho_{0}} \frac{1}{2}\left\|\Gamma\left(\rho_{0}\right)-r_{\tau}\right\|_{1}$, where $r_{\tau}$ is the desired final state. This implies

$$
\mathcal{T}\left(\rho_{\tau}, r_{\tau}\right) \leqslant \epsilon \quad \text { for all } \rho_{0}
$$

Invoking the triangle inequality, we note that

$$
\mathcal{T}\left(\rho_{\tau}, \alpha_{\tau}\right) \leqslant 2 \epsilon^{\prime} \leqslant 2 \epsilon
$$

The authors of Ref. [16, Theorem 3] provided a general bound on relative entropy between two density matrices which, when applied to $\rho_{\tau}$ and $\alpha_{\tau}$, says

$$
\begin{aligned}
D\left[\rho_{\tau} \| \alpha_{\tau}\right] \leqslant & 2 \mathcal{T}\left(\rho_{\tau}, \alpha_{\tau}\right) \ln d-2 \mathcal{T}\left(\rho_{\tau}, \alpha_{\tau}\right) \\
& \times \ln \left[2 \mathcal{T}\left(\rho_{\tau}, \alpha_{\tau}\right)\right]-\mathcal{T}\left(\rho_{\tau}, \alpha_{\tau}\right) \ln s,
\end{aligned}
$$

where $s:=\min \Lambda_{\alpha_{\tau}}$ is the smallest of $\alpha_{\tau}$ 's eigenvalues and $d$ is the dimension of the Hilbert space.

Combining Eqs. (B3) and (B4) yields successive bounds in terms of precision and error tolerance, respectively,

$$
D\left[\rho_{\tau} \| \alpha_{\tau}\right] \leqslant 4 \epsilon^{\prime} \ln \left(\frac{d}{4 \epsilon^{\prime} \sqrt{s}}\right) \leqslant 4 \epsilon \ln \left(\frac{d}{4 \epsilon \sqrt{s}}\right)
$$

The authors of Ref. [16, Theorem 5] provided still much tighter upper bounds on relative entropy between qubit states $(d=2)$, which, when applied to $\rho_{\tau}$ and $\alpha_{\tau}$, says

$$
D\left[\rho_{\tau} \| \alpha_{\tau}\right] \leqslant\left\{\begin{array}{cc}
{\left[\mathcal{T}\left(\rho_{\tau}, \alpha_{\tau}\right)+1-s\right] \ln \left[\frac{\mathcal{T}\left(\rho_{\tau}, \alpha_{\tau}\right)+1-s}{1-s}\right]+\left[s-\mathcal{T}\left(\rho_{\tau}, \alpha_{\tau}\right)\right] \ln \left[1-\frac{\mathcal{T}\left(\rho_{\tau}, \alpha_{\tau}\right)}{s}\right]} & \text { if } \mathcal{T}\left(\rho_{\tau}, \alpha_{\tau}\right) \leqslant s, \\
\max \left\{-\ln \left[1-\mathcal{T}\left(\rho_{\tau}, \alpha_{\tau}\right)\right],\right. & \\
\left.\left[s+\mathcal{T}\left(\rho_{\tau}, \alpha_{\tau}\right)\right] \ln \left[1+\frac{\mathcal{T}\left(\rho_{\tau}, \alpha_{\tau}\right)}{s}\right]+\left[1-s-\mathcal{T}\left(\rho_{\tau}, \alpha_{\tau}\right)\right] \ln \left[1-\frac{\mathcal{T}\left(\rho_{\tau}, \alpha_{\tau}\right)}{1-s}\right]\right\} & \text { if } \mathcal{T}\left(\rho_{\tau}, \alpha_{\tau}\right) \geqslant s .
\end{array}\right.
$$

Since this upper bound is monotonically increasing with $\mathcal{T}\left(\rho_{\tau}, \alpha_{\tau}\right)$ (if $s$ is held fixed), replacing $\mathcal{T}\left(\rho_{\tau}, \alpha_{\tau}\right)$ with $\epsilon^{\prime}$ yields a slightly weaker but still valid upper bound. Replacing $\epsilon^{\prime}$ with $\epsilon$ again yields a progressively weaker but still valid upper bound. Hence, we find

$$
\begin{aligned}
D\left[\rho_{\tau} \| \alpha_{\tau}\right] \leqslant & \begin{cases}\left(s-\epsilon^{\prime}\right) \ln \left(\frac{s-\epsilon^{\prime}}{s}\right)+\left(1-s+\epsilon^{\prime}\right) \ln \left(\frac{1-s+\epsilon^{\prime}}{1-s}\right) & \text { if } \epsilon^{\prime} \leqslant s, \\
\max \left\{-\ln \left(1-\epsilon^{\prime}\right),\right. & \\
\left.\left(s+\epsilon^{\prime}\right) \ln \left(\frac{s+\epsilon^{\prime}}{s}\right)+\left(1-s-\epsilon^{\prime}\right) \ln \left(\frac{1-s-\epsilon^{\prime}}{1-s}\right)\right\} & \text { if } \epsilon^{\prime} \geqslant s,\end{cases} \\
& \leqslant \begin{cases}(s-\epsilon) \ln \left(\frac{s-\epsilon}{s}\right)+(1-s+\epsilon) \ln \left(\frac{1-s+\epsilon}{1-s}\right) & \text { if } \epsilon \leqslant s, \\
\max \{-\ln (1-\epsilon), & \text { if } \epsilon \geqslant s .\end{cases}
\end{aligned}
$$

These bounds are significantly tighter than Eq. (B5). Notice that Eqs. (B7) and (B8) are convex in each region where they are a smooth function of $\epsilon^{\prime \prime} \in\left\{\epsilon, \epsilon^{\prime}\right\}$ whereas Eq. (B5) is a concave function of $\epsilon^{\prime \prime}$.

The authors of Ref. [16, Theorem 6] furthermore provided sharp upper bounds on the relative entropy between qudit states $(d>2)$, which, when applied to $\rho_{\tau}$ and $\alpha_{\tau}$, says

$$
D\left[\rho_{\tau} \| \alpha_{\tau}\right] \leqslant \begin{cases}{\left[s+\mathcal{T}\left(\rho_{\tau}, \alpha_{\tau}\right)\right] \ln \left[\frac{s+\mathcal{T}\left(\rho_{\tau}, \alpha_{\tau}\right)}{s}\right]+\left[s-\mathcal{T}\left(\rho_{\tau}, \alpha_{\tau}\right)\right] \ln \left[\frac{s-\mathcal{T}\left(\rho_{\tau}, \alpha_{\tau}\right)}{s}\right]} & \text { if } \mathcal{T}\left(\rho_{\tau}, \alpha_{\tau}\right) \leqslant s, \\ {\left[s+\mathcal{T}\left(\rho_{\tau}, \alpha_{\tau}\right)\right] \ln \left[\frac{s+\mathcal{T}\left(\rho_{\tau}, \alpha_{\tau}\right)}{s}\right]} & \text { if } s \leqslant \mathcal{T}\left(\rho_{\tau}, \alpha_{\tau}\right) \leqslant 1-s\end{cases}
$$

Again, these bounds are monotonically increasing with $\mathcal{T}\left(\rho_{\tau}, \alpha_{\tau}\right)$ (if $s$ is held fixed), so replacing $\mathcal{T}\left(\rho_{\tau}, \alpha_{\tau}\right)$ with $\epsilon^{\prime}$ or $\epsilon$ yields valid but progressively weaker upper bounds:

$$
\begin{aligned}
D\left[\rho_{\tau} \| \alpha_{\tau}\right] & \leqslant \begin{cases}\left(s+\epsilon^{\prime}\right) \ln \left(\frac{s+\epsilon^{\prime}}{s}\right)+\left(s-\epsilon^{\prime}\right) \ln \left(\frac{s-\epsilon^{\prime}}{s}\right) & \text { if } \epsilon^{\prime} \leqslant s, \\
\left(s+\epsilon^{\prime}\right) \ln \left(\frac{s+\epsilon^{\prime}}{s}\right) & \text { if } s \leqslant \epsilon^{\prime} \leqslant 1-s,\end{cases} \\
& \leqslant \begin{cases}(s+\epsilon) \ln \left(\frac{s+\epsilon}{s}\right)+(s-\epsilon) \ln \left(\frac{s-\epsilon}{s}\right) & \text { if } \epsilon \leqslant s, \\
(s+\epsilon) \ln \left(\frac{s+\epsilon}{s}\right) & \text { if } s \leqslant \epsilon \leqslant 1-s .\end{cases}
\end{aligned}
$$

These bounds show that $D\left[\rho_{\tau} \| \alpha_{\tau}\right] \rightarrow 0$ rather quickly as $\epsilon^{\prime \prime} \rightarrow 0$, for either $\epsilon^{\prime \prime} \in\left\{\epsilon, \epsilon^{\prime}\right\}$. For $\epsilon^{\prime \prime} \ll s$, the upper bound is parabolic: $D\left[\rho_{\tau} \| \alpha_{\tau}\right] \lesssim \epsilon^{\prime \prime 2} / s$. 
It is interesting to consider how these bounds apply to the examples for RESET given in the main text. For the first example, reset via SWAP, where we throw away the system state and replace it with a nearly pure state from an effectively cold environment, the final state is always given exactly by the initial bath state $\gamma$. This implies $\epsilon^{\prime}=0$ and so $\Sigma_{\rho_{0}}=k_{\mathrm{B}} D\left[\rho_{0} \| \alpha_{0}\right]$ exactly for this example, which was indeed verified by explicit calculation.

For the examples or RESET via time-dependent Lindbladian dynamics, the precision of final states is related to the eigenvalues of the Lindbladian throughout the protocol. At each moment, the Lindbladian dynamics imply an exponential decay of distinction among states, as all states converge towards the instantaneous steady state of the Lindblad operator. The decay rates are given by the nonzero eigenvalues of the Lindblad operator. In these examples, precision can always be increased by extending the duration of the protocol, and $\epsilon^{\prime}$ decays exponentially towards 0 with time. This means that $\Sigma_{\rho_{0}}=k_{\mathrm{B}} D\left[\rho_{0} \| \alpha_{0}\right]$ will be an excellent approximation for RESET via Lindbladian dynamics, given that the protocol is run for long enough to ensure reliable reset towards the desired state. Indeed, our numerical investigations verify this excellent agreement with Eq. (11).

\section{APPENDIX C: ANY RELIABLE RESET OPERATION HAS A UNIQUE MINIMALLY DISSIPATIVE STATE WITH FULL SUPPORT}

We will prove that the minimally dissipative input state to any reliable reset protocol will have full support on the domain of states to be reset.

Consider an initial state $\xi_{0}$ that does not have full support. This state has a collection of eigenvalues $\Lambda$ and a spectral decomposition $\xi_{0}=\sum_{\lambda \in \Lambda} \lambda|\lambda\rangle\langle\lambda|$ with $0 \leqslant \lambda \leqslant 1$ for all $\lambda$ and $\sum_{\lambda \in \Lambda} \lambda=1$. By assumption, $\xi_{0}$ must have a spectral decomposition with at least one zero eigenvalue with associated spectral projection operator $|o\rangle\langle o|$ that acts on a subspace of the domain to be reset. (We use $|o\rangle$ here for the eigenstate associated with the eigenvalue of zero, so that it is not confused with a computational-basis state.)

We will now show that (if entropy flow is finite for all inputs) there is always a state $\xi_{0}^{\prime}=(1-\delta) \xi_{0}+\delta|o\rangle\langle o|$ with greater support than $\xi_{0}$ that dissipates less than $\xi_{0}$ for some $0<\delta<1$. Accordingly, a state that lacks full support will never be the minimally dissipative input to a reliable reset protocol.

Recall from Eq. (17) that the entropy production during any reliable reset protocol is

$$
\Sigma_{\rho_{0}}=\boldsymbol{\Phi}_{\rho_{0}}+k_{\mathrm{B}} S\left(r_{\tau}\right)-k_{\mathrm{B}} S\left(\rho_{0}\right),
$$

where $r_{\tau}$ is the input-independent final state. Furthermore, recall from Sec. A that the expected entropy flow is a linear function of the initial state. Note that the eigenvalues of $\xi_{0}^{\prime}$ are $(1-\delta) \lambda$ for each $\lambda \in \Lambda \backslash\{0\}$, while one of the zero eigenvalues of $\xi_{0}$ maps to an eigenvalue of $\delta$ for $\xi_{0}^{\prime}$.

The entropy production from input $\xi_{0}^{\prime}$ is thus

$$
\begin{aligned}
\boldsymbol{\Sigma}_{\xi_{0}^{\prime}} & =\boldsymbol{\Sigma}_{(1-\delta) \xi_{0}+\delta|o\rangle\langle o|} \\
& =\delta \boldsymbol{\Phi}_{|o\rangle\langle o|}+(1-\delta) \boldsymbol{\Phi}_{\xi_{0}}+k_{\mathrm{B}}\left\{(1-\delta) \sum_{\lambda \in \Lambda} \lambda \ln [\lambda(1-\delta)]\right\}+k_{\mathrm{B}} \delta \ln \delta+k_{\mathrm{B}} S\left(r_{\tau}\right) \\
& =\delta \boldsymbol{\Phi}_{|o\rangle\langle o|}+(1-\delta) \boldsymbol{\Phi}_{\xi_{0}}-k_{\mathrm{B}}(1-\delta) S\left(\xi_{0}\right)+k_{\mathrm{B}}(1-\delta) \ln (1-\delta)+k_{\mathrm{B}} \delta \ln \delta+k_{\mathrm{B}} S\left(r_{\tau}\right) \\
& =\delta \boldsymbol{\Sigma}_{|o\rangle\langle o|}+(1-\delta) \boldsymbol{\Sigma}_{\xi_{0}}-k_{\mathrm{B}} \mathrm{B}(\delta),
\end{aligned}
$$

where $\mathrm{B}(\delta)=-\delta \ln \delta-(1-\delta) \ln (1-\delta)$ is the binary entropy function, and we used the fact that $\sum_{\lambda \in \Lambda} \lambda=1$.

If the difference between $\boldsymbol{\Sigma}_{\xi_{0}}$ and $\boldsymbol{\Sigma}_{\xi_{0}^{\prime}}$ is positive, then $\xi_{0}$ cannot be a minimally dissipative state. From Eq. (C4), we find

$$
\boldsymbol{\Sigma}_{\xi_{0}}-\boldsymbol{\Sigma}_{\xi_{0}^{\prime}}=k_{\mathrm{B}} \mathrm{B}(\delta)-\delta\left(\boldsymbol{\Sigma}_{|o\rangle\langle o|}-\boldsymbol{\Sigma}_{\xi_{0}}\right) .
$$

If $\delta=0$ then, of course, $\Sigma_{\xi_{0}}=\Sigma_{\xi_{0}^{\prime}}$ since $\xi_{0}^{\prime}$ is then, in fact, equal to $\xi_{0}$. However, we find that $\boldsymbol{\Sigma}_{\xi_{0}}-\boldsymbol{\Sigma}_{\xi_{0}^{\prime}}$ has a positive derivative with respect to $\delta$, from $\delta=0$ up to some sufficiently small finite value. That is,

$$
\frac{d}{d \delta}\left(\boldsymbol{\Sigma}_{\xi_{0}}-\boldsymbol{\Sigma}_{\xi_{0}^{\prime}}\right)=k_{\mathrm{B}} \ln \left(\frac{1-\delta}{\delta}\right)-\left(\boldsymbol{\Sigma}_{|\rho\rangle\langle\rho|}-\boldsymbol{\Sigma}_{\xi_{0}}\right) .
$$

Since $\boldsymbol{\Sigma}_{|o\rangle\langle o|}-\boldsymbol{\Sigma}_{\xi_{0}}$ is assumed to be finite, while $\ln \left(\frac{1-\delta}{\delta}\right)$ grows unbounded as $\delta \rightarrow 0$, this tells us that $\boldsymbol{\Sigma}_{\xi_{0}}>\boldsymbol{\Sigma}_{\xi_{0}^{\prime}}$ for sufficiently small $\delta$. Hence, $\xi_{0}$ cannot be the minimally dissipative state of any reliable reset protocol. Notably, the only assumption that we made about $\xi_{0}$ is that it does not have full support.
We showed that any state lacking full support cannot be the minimally dissipative input for any reliable reset protocol. Thus, the minimally dissipative state $\alpha_{0}$ must have full support on the domain of states to be reset.

By Eq. (11), the dissipation from any input state $\rho_{0}$ exceeds this minimal dissipation by $k_{\mathrm{B}} D\left[\rho_{0} \| \alpha_{0}\right]$, which is positive for all $\rho_{0} \neq \alpha_{0}$.

\section{APPENDIX D: EXISTENCE OF (AND AN EXPRESSION FOR) THE ENTROPY FLOW VECTOR}

Entropy flow is an affine function of the initial state. Recall from Eq. (5) that the expected entropy flow can be expressed as

$$
\begin{aligned}
\boldsymbol{\Phi}_{\rho_{0}} & =-k_{\mathrm{B}} \int_{0}^{\tau} \operatorname{tr}\left(\dot{\rho}_{t}^{\mathrm{env}} \ln \pi_{x_{t}}^{\mathrm{env}}\right) d t \\
& =-k_{\mathrm{B}} \int_{0}^{\tau} \operatorname{tr}\left\{\left[\frac{d}{d t} \operatorname{tr}_{\text {sys }}\left(\mathcal{U}_{x_{0: t}} \rho_{0} \otimes \rho_{0}^{\mathrm{env}} \mathcal{U}_{x_{0: t}^{\dagger}}^{\dagger}\right)\right] \ln \pi_{x_{t}}^{\mathrm{env}}\right\} d t,
\end{aligned}
$$


where the environmental reference state $\pi_{x_{t}}^{\text {env }}$ is independent of the initial state of the system. that

For any initial state $\rho_{0}=I / 2+\frac{1}{2} \vec{a} \cdot \vec{\sigma}$, it is then clear

$$
\begin{aligned}
\boldsymbol{\Phi}_{\rho_{0}} & =-k_{\mathrm{B}} \int_{0}^{\tau} \operatorname{tr}\left(\left\{\frac{d}{d t} \operatorname{tr} \text { sys }\left[\mathcal{U}_{x_{0: t}}\left(I / 2+\frac{1}{2} \vec{a} \cdot \vec{\sigma}\right) \otimes \rho_{0}^{\mathrm{env}} \mathcal{U}_{x_{0: t}}^{\dagger}\right]\right\} \ln \pi_{x_{t}}^{\mathrm{env}}\right) d t \\
& =\boldsymbol{\Phi}_{I / 2}-\frac{1}{2} \vec{a} \cdot k_{\mathrm{B}} \int_{0}^{\tau} \operatorname{tr}\left\{\left[\frac{d}{d t} \operatorname{tr}_{\text {sys }}\left(\mathcal{U}_{x_{0: t}} \vec{\sigma} \otimes \rho_{0}^{\mathrm{env}} \mathcal{U}_{x_{0: t}^{\dagger}}^{\dagger}\right)\right] \ln \pi_{x_{t}}^{\mathrm{env}}\right\} d t .
\end{aligned}
$$

This shows that there exists an initial-state-independent vector $\vec{\phi}=\left(\phi_{x}, \phi_{y}, \phi_{z}\right)$ such that

$$
\boldsymbol{\Phi}_{\rho_{0}}=\boldsymbol{\Phi}_{I / 2}+\frac{1}{2} \vec{a} \cdot \vec{\phi}
$$

We call $\vec{\phi}$ the "entropy-flow vector" induced by the control protocol.

Beyond merely proving its existence, Eq. (D3) gives an explicit construction for the entropy-flow vector

$$
\vec{\phi}=-k_{\mathrm{B}} \int_{0}^{\tau} \operatorname{tr}\left\{\left[\frac{d}{d t} \operatorname{tr}_{\text {sys }}\left(\mathcal{U}_{x_{0: t}} \vec{\sigma} \otimes \rho_{0}^{\mathrm{env}} \mathcal{U}_{x_{0: t}^{\dagger}}^{\dagger}\right)\right] \ln \pi_{x_{t}}^{\mathrm{env}}\right\} d t .
$$

Alternatively, this could be written component-wise as

$$
\phi_{k}=-k_{\mathrm{B}} \int_{0}^{\tau} \operatorname{tr}\left\{\left[\frac{d}{d t} \operatorname{tr}_{\text {sys }}\left(\mathcal{U}_{x_{0: t}} \sigma_{k} \otimes \rho_{0}^{\mathrm{env}} \mathcal{U}_{x_{0: t}^{\dagger}}^{\dagger}\right)\right] \ln \pi_{x_{t}}^{\mathrm{env}}\right\} d t
$$

for $k \in\{x, y, z\}$.

When the environmental reference state is time independent, such that $\pi_{x_{t}}^{\text {env }}=\bigotimes_{b \in \mathbb{B}} \pi^{(b)}$, this reduces to

$$
\vec{\phi}=-\sum_{b \in \mathbb{B}} k_{\mathrm{B}} \operatorname{tr}\left[\operatorname{tr}_{\mathrm{sys}, \mathbb{B} \backslash b}\left(\mathcal{U}_{x_{0: \tau}} \vec{\sigma} \otimes \rho_{0}^{\mathrm{env}} \mathcal{U}_{x_{0: \tau}}^{\dagger}\right) \ln \pi^{(b)}\right] .
$$

\section{APPENDIX E: FURTHER IMPLEMENTATIONS FOR QUANTUM MEMORY RESET IN THE WEAK COUPLING REGIME}

Here we discuss an approach to memory reset in the weak coupling regime.

We first consider the simplest scenario of single qubit reset to $|0\rangle$. An obvious way to implement reset is the following.

(1) Attach the qubit to a single thermal bath at temperature $T$.

(2) Change the system Hamiltonian from $H_{x_{0}}$ to $H_{x_{\tau}}=$ $E|1\rangle\langle 1|$ with $E \gg k_{\mathrm{B}} T$.

(3) Allow the system to equilibrate, so that $\rho_{\tau} \approx \boldsymbol{\pi}_{x_{\tau}} \approx$ $|0\rangle\langle 0|$.

If the desired reset state is a pure state $r_{\tau} \neq|0\rangle\langle 0|$, then the system can subsequently be detached from the thermal reservoir and unitarily evolved to $r_{\tau}$. Alternatively, the desired state can be attained more directly by driving to the final Hamiltonian $H_{x_{\tau}}=E\left(I-r_{\tau}\right)$. With the luxury of much time, the error can be made arbitrarily small.

A related procedure allows reliable reset to any state $r_{\tau}$.
(1) Attach the qubit to a single thermal bath at temperature $T$.

(2) Change the system Hamiltonian from $H_{x_{0}}$ to $H_{x_{\tau}}=$ $-k_{\mathrm{B}} T \ln r_{\tau}+c$, where $c$ is an arbitrary constant.

(3) Allow the system to equilibrate to $\pi_{x_{\tau}}=r_{\tau}$.

In the quasistatic limit, this reset can be achieved with zero entropy production if the initial state is known. The protocol can then be designed to make $\rho_{0}=\alpha_{0}$. To achieve zero dissipation, the initial system-Hamiltonian should change instantaneously from $H_{x_{0}}$ to $H_{x_{0^{+}}}=-k_{\mathrm{B}} T \ln \rho_{0}+c^{\prime}$, where $c^{\prime}$ is an arbitrary constant. Subsequently, the Hamiltonian should be changed quasistatically (via any continuous path) from $H_{x_{0}+}$ to $H_{x_{\tau}}$. In such scenarios, the work invested will equal the change in free energy, so that there is zero entropy production. If, however, the initial state $\rho_{0}$ is not equal to the anticipated state $\alpha_{0}=\pi_{x_{0}+} \propto e^{-\beta H_{x_{0}}}$, then there will be dissipation equal to the loss of initially induced nonequilibrium addition to free energy: $\Sigma_{\rho_{0}}=k_{\mathrm{B}} D\left[\rho_{0} \| \pi_{x_{0^{+}}}\right]$.

Notably, in this sudden-then-quasistatic regime, the fully mixed state can be designed to be the minimally dissipative state by choosing $H_{x_{0+}}$ to be a fully degenerate Hamiltonian. The entropy production will then be $\Sigma_{\rho_{0}}=k_{\mathrm{B}} D\left[\rho_{0} \| I / d\right]$ for a $d$-dimensional system. As mentioned in the main text, this results in a constant heat $Q=k_{\mathrm{B}} T \ln d$, independent of the input. While this heat is relatively small, it is, however, larger than Landauer's bound whenever $\rho_{0} \neq I / d$.

In the slow but finite-duration linear response regime, reliable reset can still be achieved within some small error tolerance $\epsilon$, but the dissipation will scale as $1 / \tau$. Optimal protocols in the linear response regime can be found as natural geodesics induced by the friction tensor [26-28].

As the protocol becomes quick relative to the relaxation timescales for the system, the protocol must be modified to maintain reliable reset to the desired state. One way to achieve this is via counterdiabatic alterations to the driving [29]. Counterdiabatic driving can, for example, enforce that the system stays along the optimal linear-response state trajectory.

In these finite-time scenarios, the dissipation will be nontrivial for all initial states, but our equality relating dissipation among all possible initial states will remain valid.

These results can be easily extended to a scenario for quantum reset of $N$ qubits. An obvious way to implement RESET is the following.

(1) Attach the $N$ qubits to a single thermal bath at temperature $T$.

(2) Change the system's Hamiltonian from $H_{x_{0}}$ to $H_{x_{\tau}}=$ $E\left(I-(|0\rangle\langle 0|)^{\otimes N}\right)$ with $E \gg k_{\mathrm{B}} T$. 
(3) Allow the system to equilibrate, so that $\rho_{\tau} \approx \pi_{x_{\tau}} \approx$ $(|0\rangle\langle 0|)^{\otimes N}$.

(4) Detach the qubits from the thermal bath and unitarily transform them to any desired $N$-qubit pure state.

This allows, for example, reset to the maximally entangled state. Alternatively, the desired reset state could be achieved more directly by designing the final Hamiltonian $H_{x_{\tau}}$ such that $r_{\tau}$ is its equilibrium state. This suggests the alternative procedure for resetting to any $N$-qubit state.
(1) Attach the $N$ qubits to a single thermal bath at temperature $T$.

(2) Change the system's Hamiltonian from $H_{x_{0}}$ to $H_{x_{\tau}}=$ $-k_{\mathrm{B}} T \ln r_{\tau}+c$, where $c$ is an arbitrary constant.

(3) Allow the system to equilibrate to $\pi_{x_{\tau}}=r_{\tau}$.

The earlier comments about finite-time protocols (linear response, counterdiabatic driving, etc.) all apply to this general $N$-qubit reset as well.
[1] R. Landauer, Irreversibility and heat generation in the computing process, IBM J. Res. Dev. 5, 183 (1961).

[2] J. M. R. Parrondo, J. M. Horowitz, and T. Sagawa, Thermodynamics of information, Nat. Phys. 11, 131 (2015).

[3] R. Alicki, M. Horodecki, P. Horodecki, and R. Horodecki, Thermodynamics of quantum information systems-Hamiltonian description, Open Syst. Inf. Dyn. 11, 205 (2004).

[4] D. Reeb and M. M. Wolf, An improved Landauer principle with finite-size corrections, New J. Phys. 16, 103011 (2014).

[5] Y. Jun, M. Gavrilov, and J. Bechhoefer, High-Precision Test of Landauer's Principle, Phys. Rev. Lett. 113, 190601 (2014).

[6] H. J. D. Miller, G. Guarnieri, M. T. Mitchison, and J. Goold, Quantum Fluctuations Hinder Finite-Time Information Erasure Near the Landauer Limit, Phys. Rev. Lett. 125, 160602 (2020).

[7] P. M. Riechers and M. Gu, Initial-state dependence of thermodynamic dissipation for any quantum process, Phys. Rev. E 103, 042145 (2021).

[8] O. C. O. Dahlsten, R. Renner, E. Rieper, and V. Vedral, Inadequacy of von Neumann entropy for characterizing extractable work, New J. Phys. 13, 053015 (2011).

[9] N. Y. Halpern, A. J. P. Garner, O. C. O. Dahlsten, and V. Vedral, Introducing one-shot work into fluctuation relations, New J. Phys. 17, 095003 (2015).

[10] M. Esposito, K. Lindenberg, and C. Van den Broeck, Entropy production as correlation between system and reservoir, New J. Phys. 12, 013013 (2010).

[11] K. Ptaszyński and M. Esposito, Entropy Production in Open Systems: The Predominant Role of Intraenvironment Correlations, Phys. Rev. Lett. 123, 200603 (2019).

[12] R. Clausius, On a modified form of the second fundamental theorem in the mechanical theory of heat, The London, Edinburgh, and Dublin Phil. Magazine J. Sci. 12, 81 (1856).

[13] S. R. de Groot and P. Mazur, Non-Equilibrium Thermodynamics (Dover, New York, 1984).

[14] D. Kondepudi and I. Prigogine, Modern Thermodynamics: From Heat Eengines to Dissipative Structures (John Wiley \& Sons, New York, 2014).
[15] A. Kolchinsky and D. H. Wolpert, Dependence of dissipation on the initial distribution over states, J. Stat. Mech. (2017) 083202.

[16] K. M. R. Audenaert and J. Eisert, Continuity bounds on the quantum relative entropy, J. Math. Phys. 46, 102104 (2005).

[17] T. Baumgratz, M. Cramer, and M. B. Plenio, Quantifying Coherence, Phys. Rev. Lett. 113, 140401 (2014).

[18] M. Lostaglio, D. Jennings, and T. Rudolph, Description of quantum coherence in thermodynamic processes requires constraints beyond free energy, Nat. Commun. 6, 6383 (2015).

[19] G. Francica, J. Goold, and F. Plastina, Role of coherence in the nonequilibrium thermodynamics of quantum systems, Phys. Rev. E 99, 042105 (2019).

[20] J. P. Santos, L. C. Céleri, G. T. Landi, and M. Paternostro, The role of quantum coherence in non-equilibrium entropy production, npj Quant. Info. 5, 23 (2019).

[21] G. Manzano, J. M. Horowitz, and J. M. R. Parrondo, Nonequilibrium potential and fluctuation theorems for quantum maps, Phys. Rev. E 92, 032129 (2015).

[22] A. J. Leggett, S. Chakravarty, A. T. Dorsey, M. P. A. Fisher, A. Garg, and W. Zwerger, Dynamics of the dissipative two-state system, Rev. Mod. Phys. 59, 1 (1987).

[23] G. W. Wimsatt, A. B. Boyd, P. M. Riechers, and J. P. Crutchfield, Refining Landauer's stack: Balancing error and dissipation when erasing information, J. Stat. Phys. 183, 16 (2021).

[24] L. E. Reichl, A Modern Course in Statistical Physics (WileyVCH, Weinham, Germany, 2009).

[25] R. A. Alberty, Use of Legendre transforms in chemical thermodynamics (IUPAC technical report), Pure Appl. Chem. 73, 1349 (2001).

[26] D. A. Sivak and G. E. Crooks, Thermodynamic Metrics and Optimal Paths, Phys. Rev. Lett. 108, 190602 (2012).

[27] D. Mandal and C. Jarzynski, Analysis of slow transitions between nonequilibrium steady states, J. Stat. Mech. (2016) 063204.

[28] M. Scandi and M. Perarnau-Llobet, Thermodynamic length in open quantum systems, Quantum 3, 197 (2019).

[29] G. Vacanti, R. Fazio, S. Montangero, G. M. Palma, M. Paternostro, and V. Vedral, Transitionless quantum driving in open quantum systems, New J. Phys. 16, 053017 (2014). 